\title{
Activation and Functional Priming of Blood Neutrophils in Non-Alcoholic Fatty Liver Disease Increases in Non-Alcoholic Steatohepatitis
}

\author{
Johanne Sloth Lauszus' \\ Peter Lykke Eriksen (D) \\ Mette Mejlby Hansen' \\ Lotte Lindgreen Eriksen' \\ Debbie Lindsay Shawcross (iD ${ }^{2,3}$ \\ Hendrik Vilstrup iD ' \\ Karen Louise Thomsen (ID \\ Sidsel Stoy (D) \\ 'Department of Hepatology and \\ Gastroenterology, Aarhus University \\ Hospital, Aarhus, Denmark; ${ }^{2}$ Institute of \\ Liver Studies, School of Immunology and \\ Microbial Sciences, Faculty of Life \\ Sciences and Medicine, King's College \\ London, London, UK; ${ }^{3}$ Institute of Liver \\ Studies, King's College Hospital NHS \\ Foundation Trust, London, UK
}

Introduction: In non-alcoholic fatty liver disease (NAFLD), neutrophils in liver infiltrates are activated, which may contribute to disease progression towards non-alcoholic steatohepatitis (NASH). However, the functional status of the blood neutrophils remains unknown and their role in the disease mechanisms is thus uncertain. We therefore characterized activation and function of blood neutrophils in patients with NAFLD in relation to clinical disease markers and the NAFLD plasma milieu.

Methods: We studied 20 patients with NAFLD, among these 6 patients with NASH, and 14 healthy persons. Neutrophil activation, interleukin (IL)-8 production and oxidative burst were measured by flow cytometry on participants' neutrophils and on healthy neutrophils exposed in vitro to plasma from the study participants.

Results: Blood neutrophils from the NASH patients showed a doubling in their expression of the activation marker CD62L. Also, all NAFLD patients had 50-100\% increased expression of CD11b. Functionally, NASH neutrophils had 30\% elevated IL-8 production and more than doubled spontaneous oxidative burst. In all NAFLD patients, higher spontaneous oxidative burst was associated with worse liver function. Incubation of healthy neutrophils with NAFLD plasma paradoxically slightly reduced CD62L and CD11b expression, and NASH plasma also reduced the frequency of IL-8-producing neutrophils.

Conclusion: In NAFLD, blood neutrophils are activated, and in NASH also functionally primed. This suggests a progressive neutrophil aggressiveness already present with liver fat infiltration. However, NAFLD plasma in vitro, if anything, had the opposite effect on the healthy neutrophils so the NAFLD-related neutrophil activation cannot be attributed to humoral factors and remains unexplained.

Keywords: neutrophil, non-alcoholic fatty liver disease, non-alcoholic steatohepatitis, oxidative burst, CD11b

\section{Introduction}

Non-alcoholic fatty liver disease (NAFLD) is strongly associated with the metabolic syndrome and is now the most common chronic liver disease in most countries. $^{1-3}$ Chronic, low-grade, systemic inflammation is an established feature of NAFLD, but it remains poorly understood how the immune activation evolves with disease progression from simple steatosis, non-alcoholic fatty liver (NAFL), to non-alcoholic steatohepatitis (NASH) which carries a risk of fibrosis and ultimately cirrhosis. However, it is known that in NASH patients, neutrophils are highly abundant in the liver and also in the blood. ${ }^{4}$ Likewise, a high blood neutrophil-tolymphocyte ratio is a prognostic marker for development of NASH and fibrosis. ${ }^{5}$
Correspondence: Sidsel Stoy

Department of Hepatology and

Gastroenterology, Aarhus University

Hospital, Palle Juul Jensens Boulevard 99,

Aarhus, Denmark

Email Sidsel_stoy@clin.au.dk 
Still, functional studies of neutrophils in human NAFLD are scarce, particularly in regards to blood neutrophils. In general, blood bacterial peptides or inflammatory mediators such as complement factors activate neutrophils by binding to their surface receptors in the blood stream. This upregulates integrin receptors such as $\mathrm{CD} 11 \mathrm{~b}$ and $\mathrm{CD} 62 \mathrm{~L}$, which initiates neutrophil migration from blood to tissues. Activated neutrophils furthermore produce the chemotactic cytokine interleukin (IL)-8 that recruits and activates additional neutrophils. Neutrophil activation involves expression of myeloid peroxidase to induce the generation of reactive oxygen species (ROS) enabling neutrophil oxidative burst, which is the essential killing mechanism of neutrophils. ${ }^{6}$

In alcohol-related cirrhosis, blood neutrophils are functionally primed with an elevated spontaneous oxidative burst and impaired phagocytosis, both of which are associated with disease progression and infections. ${ }^{7,8}$ This neutrophil activation can be induced by humoral factors in blood such as endotoxins, since incubation of healthy neutrophils with plasma from patients replicates the patient neutrophil phenotype. ${ }^{9}$ A study of NASH patients found an increased oxidative burst of blood neutrophils following stimulation with phorbol 12-myristate 13acetate (PMA). ${ }^{10}$ On this basis, we hypothesized that blood neutrophils in NAFLD are activated and functionally primed, that this is more marked in the patients with NASH and that this neutrophil phenotype develops in response to blood humoral factors and therefore is transmissible to healthy neutrophils via patient plasma.

The aims of the present study were therefore, firstly, to examine the activation and functional status of blood neutrophils in NAFL and NASH patients, secondly to investigate how this relates to clinical markers of disease stage, and lastly to determine whether a NAFL- or NASH-related neutrophil phenotype and dysfunction can be elicited by the patients' plasma.

\section{Methods}

\section{Patients}

Twenty patients with non-diabetic NAFLD (14 with NAFL and 6 with NASH; 6 female, median age of 39 years (range, 18-60 years)) were consecutively recruited from the Department of Hepatology and Gastroenterology, Aarhus University Hospital, Central Denmark Region, in this crosssectional study. The exclusion criteria and the diagnosis of biopsy-proven NAFLD were as previously published. ${ }^{11}$ The metabolic profile and the liver and inflammatory status of the patients were described by biochemical markers. The aminopyrine breath test was used to assess the metabolic liver function and was conducted on 14 patients including all NASH patients and in 10/14 healthy persons. It is expressed as percentage dose recovery (PDR) peak $(\% / \mathrm{h})$ as previously described. ${ }^{11}$ The 14 controls were healthy, age- and sexmatched persons (5 female, median age 37 (range 22-61)) with BMI $<25 \mathrm{~kg} / \mathrm{m}^{2}$ and no clinical signs or histories of liver disease. Plasma from 10 controls (7 male, median age of 36 years (range, 22-61 years)) was available for the in vitro stimulation experiment. All participants provided written informed consent prior to study inclusion and the study was conducted in accordance with the Helsinki Declaration. The study was approved by the Central Denmark Region Ethics Committee (1-10-72-140-14) and the Danish Data Protection Agency (1-16-02-322-15).

\section{Sample Collection}

Blood was collected from the fasting study participants in heparin and EDTA vacuum tubes. Standard biochemical analyses were performed by routine analysis using accredited laboratory assays at the Department of Clinical Biochemistry, Aarhus University Hospital. For the neutrophil assays, the heparinized blood samples were kept on ice and the assays started within 30 minutes of the bleed. The EDTA tubes were centrifuged for 10 minutes and the plasma was stored at $-80{ }^{\circ} \mathrm{C}$ for later analysis.

\section{Neutrophil Phenotyping and Function}

Surface staining of neutrophils was performed on whole blood with optimized amounts of fluorochrome-conjugated antibodies -C D16-APC, CD11b-APC-CY7, CD62LFITC, TLR4-Biotin (Becton Dickinson (BD), San Diego, CA, USA) - following lysis of erythrocytes with FACS lysis solution (BD, USA). All samples were analyzed immediately after preparation. To detect IL-8, the neutrophils were permeabilized with Cytofix-Cytoperm (BD, USA) following surface staining with CD16 and CD11b. Following washing with Perm Wash (BD, UK), the cells were stained intracellularly with IL-8-PE (BD, USA) and immediately analyzed on the flow cytometer. Neutrophil oxidative burst was measured by the PhagoBurst kit (Orpegen Pharma, Heidelberg, Germany) following the manufacturer's instructions. Oxidative burst is reported in unstimulated neutrophils (spontaneous oxidative burst), as well as after stimulation with formyl-Met-Leu-Phe (fMLP) (low burst), PMA (high burst), or opsonized E. coli 
(phagoburst) as previously published. ${ }^{12}$ All patient samples were run in triplicates and the median of the triplicates reported. All patient neutrophil assays were analyzed on a FACS Canto II analyzer (BD, USA).

\section{Healthy Neutrophil Incubation with Study Participants' Plasma}

Blood from a healthy, non-smoking volunteer with normal neutrophil counts and no strenuous exercise or alcohol intake in 2 days prior to the bleed was drawn into heparin-coated tubes, and the neutrophils were isolated by polymorphprep (Alere A/S, Denmark) as previously published. ${ }^{13}$ From each study participant (NAFL, NASH and healthy controls), $100 \mu \mathrm{L}$ plasma was added to $300 \mu \mathrm{L}$ healthy neutrophil suspension and incubated at $37^{\circ} \mathrm{C}$ for 2 hours. For IL-8 detection, $2 \mu \mathrm{L}$ 1:10 diluted GolgiStop was added during incubation. The neutrophils were harvested and stained for CD11b, CD62L, CD16, TLR4 and IL-8 as described above. To examine the plasma-induced burst, $20 \mu \mathrm{L}$ rhodamine was added and following incubation for 20 minutes at $37{ }^{\circ} \mathrm{C}$, washing and centrifuging, the samples were resuspended in PBS. The samples were all analyzed immediately after preparation on a MACS Quant Analyzer (Miltenyi Biotec, Bergisch Gladbach, Germany). Unstained samples and fluorescence minus one were included as controls.

\section{Data Analyses and Statistics}

Data analysis was conducted in FlowJo (Treestar Inc, Ashland, OR, USA) and reported as percentage positive cells or median fluorescence intensity (MFI). Statistical analyses were performed in Stata Version 14.2 (StataCorp, TX, USA) and figures designed in GraphPad Prism version 9 (GraphPad Software, CA, USA). One-way analysis of variance (ANOVA) was used on normally distributed data and Kruskal-Wallis analysis for skewed data to investigate differences among the groups: NAFL, $\mathrm{NASH}$, and healthy persons. An unpaired $t$-test was used for intergroup comparisons in normally distributed data, and for skewed data Mann-Whitney test was used. Fisher's exact test was used for ordinal data. Spearman's rank correlation coefficient $(\rho)$ is given for correlation analyses, which were performed on the whole NAFLD group. $\mathrm{P}<0.05$ in a two-tailed test was regarded as statistically significant.

\section{Results}

\section{Patients}

The 14 NAFL and the six NASH patients were comparable except for higher plasma alanine transaminases, triglyceride, and glucagon in the latter (Table 1). There were no differences in blood inflammatory markers such as C-reactive protein or leukocyte count. Moreover, liver biopsy inflammation scores were similar, but NASH patients were diagnosed by the presence of hepatocyte ballooning and fibrosis, all mild grade 1 fibrosis (Table 2). The aminopyrine breath test was comparable between both patient groups (PDR-peak $5.2 \pm 2 \%$ /hour vs $4.2 \pm 2.4 \%$ /hour, $\mathrm{p}=0.41$ ) but significantly lower than in the healthy persons $(7.3 \pm 1.9 \%$ /hour; $\mathrm{p}=0.04, \mathrm{p}=0.01)$.

\section{Blood Neutrophils Have a Primed Phenotype in NALFD}

In the blood, neutrophils from the NASH patients had nearly a doubling in their expression of CD62L compared with neutrophils from both NAFL and healthy persons (Figure 1A). Neutrophils from the NAFL and NASH patients on average had a $50 \%$ and $100 \%$, respectively, increased expression of the activation marker CD11b

Table I Clinical and Biochemical Characteristics of the Patients with Non-Alcoholic Fatty Liver Disease

\begin{tabular}{|c|c|c|c|}
\hline & NAFL & NASH & $\mathbf{p}$ \\
\hline & $(n=14)$ & $(n=6)$ & \\
\hline Age (years) & $40 \pm 11$ & $37 \pm 15$ & 0.6 \\
\hline Gender (n, \% female) & $5(36)$ & I (I7) & 0.6 \\
\hline $\mathrm{BMI}, \mathrm{kg} / \mathrm{m}^{2}$ & $37 \pm 8.5$ & $38 \pm 8.3$ & 0.8 \\
\hline ALT, U/I & $64 \pm 40$ & $|10 \pm 5|$ & $0.04 *$ \\
\hline Bilirubin, $\mu \mathrm{mol} / \mathrm{l}$ & $8 \pm 4.2$ & $7 \pm 2.4$ & 0.7 \\
\hline Albumin, $g / l$ & $39 \pm 1.9$ & $38 \pm 2.7$ & 0.7 \\
\hline Platelets, $10^{9} / \mathrm{I}$ & $253 \pm 59$ & $273 \pm 77$ & 0.5 \\
\hline Total cholesterol, $\mathrm{mmol} / \mathrm{l}$ & $4.5 \pm 0.8$ & $5.23 \pm 1.5$ & 0.19 \\
\hline $\mathrm{HDL}, \mathrm{mmol} / \mathrm{l}$ & $1.2 \pm 0.3$ & $1.0 \pm 0.3$ & 0.26 \\
\hline Triglyceride, $\mathrm{mmol} / \mathrm{l}$ & I.2(0.7) & $2(0.6)$ & $0.04 *$ \\
\hline Glucose, $\mathrm{mmol} / \mathrm{l}$ & $6.0 \pm 0.5$ & $6.6 \pm 0.7$ & 0.08 \\
\hline Glucagon, pmol/l & $10.4 \pm 2.0$ & $14.9 \pm 2.7$ & $0.004 *$ \\
\hline Leukocytes, $10^{9} / I$ & $7.2 \pm 2.7$ & $5.9 \pm 1.2$ & 0.3 \\
\hline Neutrophils, $10^{9} / \mathrm{l}$ & $2.99 \pm 0.80$ & $3.19 \pm 0.63$ & 0.6 \\
\hline NLR & $1.40 \pm 0.38$ & $1.72 \pm 04 \mid$ & 0.14 \\
\hline CRP, mg/l & $5.0 \pm 4.5$ & $3.7 \pm 2.9$ & 0.6 \\
\hline Soluble CDI63, mg/l & $2.2 \pm 0.6$ & $2.9 \pm 1.1$ & 0.07 \\
\hline
\end{tabular}

Notes: Estimates are given as mean \pm SD or median (IQR). Student's $t$-test or Wilcoxon rank-sum test, Fisher's exact test was used for ordinal data. *Marks p-values $<0.05$.

Abbreviations: NASH, non-alcoholic steatohepatitis; BMI, body mass index; ALT, alanine transaminase; AST, aspartate transaminase; HDL, high-density lipoprotein ; NLR, neutrophil-to-lymphocyte ratio. 
Table 2 Liver Histopathology of the Patients with Nonalcoholic Fatty Liver Disease

\begin{tabular}{|l|c|c|}
\hline & NAFL & NASH \\
\cline { 2 - 3 } & $\mathbf{( n = 1 4 )}$ & (n=6) \\
\hline Steatosis $^{\dagger}, \mathrm{n}_{1 / 2 / 3}$ & $2 / 4 / 8$ & $0 / 0 / 6$ \\
Inflammation $^{\dagger}, \mathrm{n}_{0 / 1 / 2}$ & $2 / / 2 / 0$ & $0 / 6 / 0$ \\
Ballooning $^{\dagger}, \mathrm{n}_{\mathrm{I} / 2}$ & $0 / 0$ & $4 / 2$ \\
NAS score $^{\ddagger}$ & $4(\mathrm{l}-4)$ & $5(5-6)$ \\
Fibrosis score $^{\S}$ & all 0 & all la \\
\hline
\end{tabular}

Notes: Estimates are given as number of patients except for ${ }^{\ddagger}$ where median (range) is given. ${ }^{\dagger}$ Based on steatosis activity fibrosis (SAF) score, ${ }^{\S}$ Based on Kleiner fibrosis score.

Abbreviation: NAS, non-alcoholic fatty liver disease activity score.

(Figure 1B). There was no difference in neutrophil expression of CD16 between the groups (data not shown). Similarly, there was no difference in the expression of TLR4 on neutrophils among the groups $(\mathrm{p}=0.2)$ (Figure 1C).

\section{High IL-8 and Spontaneous Oxidative Burst in Blood Neutrophils from NASH \\ Patients}

In the blood from NASH patients, the frequency of IL8-producing neutrophils was about twice that of both the NAFL patients and the healthy persons (Figure 2A). Additionally, the NASH blood neutrophils had nearly a doubling of the spontaneous oxidative burst (MFI) compared with those from NAFL patients and healthy persons.
The frequency of neutrophils with spontaneous oxidative burst was similar among the groups (Figure 2B and C). The same pattern was observed for the fMLP-induced low burst (MFI), which was higher in neutrophils from NASH patients whilst the frequency of neutrophils producing an oxidative burst in response to fMLP stimulation did not differ among the groups (Figure 2D and E). The oxidative burst in neutrophils exposed to PMA and E. coli was not different among the groups (data not shown).

\section{Higher Neutrophil Spontaneous}

\section{Oxidative Burst is Related to Worse Liver Function and Glycemic Control}

In all NAFLD patients, higher spontaneous oxidative burst MFI (PDR-peak $\rho=-0.56 ; \mathrm{p}=0.04$ ) and lower frequency of IL-8-positive neutrophils (PDR-peak $\rho=0.57 ; \mathrm{p}=0.03$ ) were associated with a lower aminopyrine breath test. Also, higher neutrophil activation as measured by CD11b MFI was associated with higher plasma triglycerides ( $\rho=0.50, \mathrm{p}=0.04$ ), and higher frequencies of IL-8-positive neutrophils were associated with higher blood glucose $(\rho=0.54, \mathrm{p}=0.02)$.

\section{NAFLD Plasma Does Not Increase but Rather Depresses Neutrophil Activation \\ and Function in vitro}

The incubation of healthy blood neutrophils with plasma from the NAFLD patients compared with healthy plasma
A

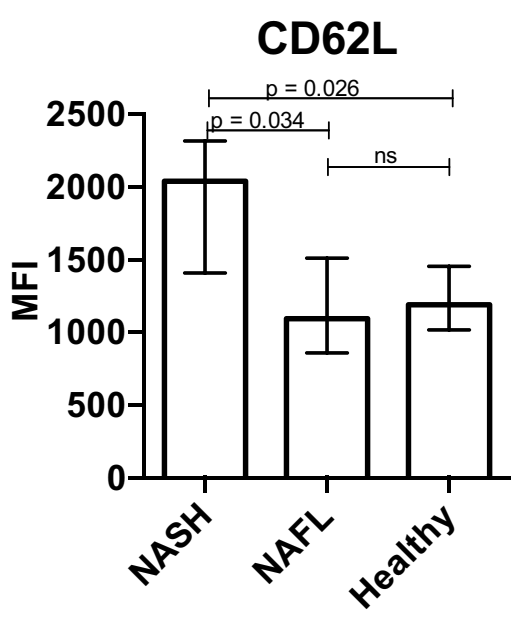

B

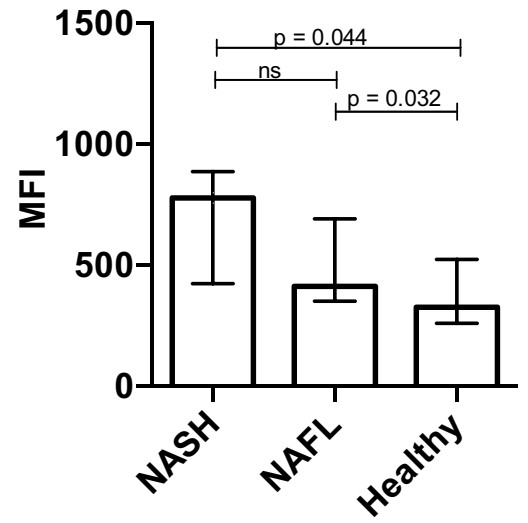

\section{CD11b}

C

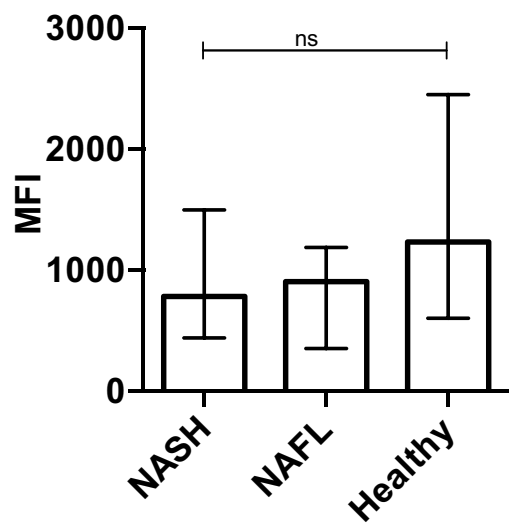

Figure I Peripheral neutrophils are activated in patients with non-alcoholic fatty liver disease. The neutrophil activation markers (A) CD62L and (B) CDI Ib and (C) the toll-like receptor (TLR) 4 were quantified on fresh neutrophils from peripheral blood by flow cytometry. Receptor expression is presented as median fluorescence intensities (MFI) of $\mathrm{CDI}^{+}$neutrophils and compared between patients with non-alcoholic steatohepatitis (NASH), non-alcoholic fatty liver (NAFL) and healthy subjects (rank sum test). Figures show median and interquartile range. 


\section{Spontaneous oxidative burst}

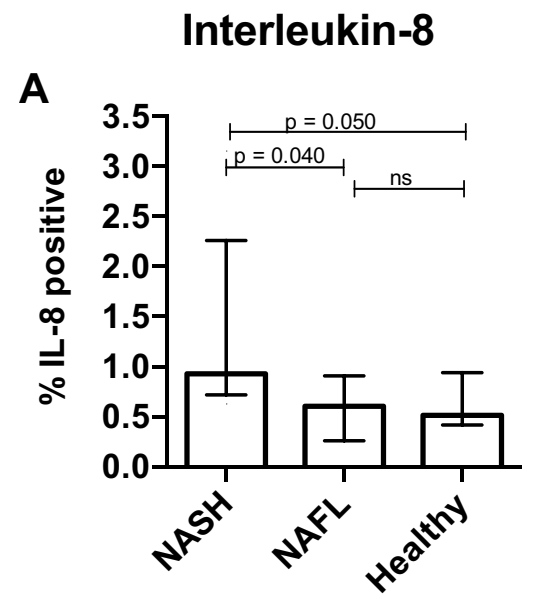

B

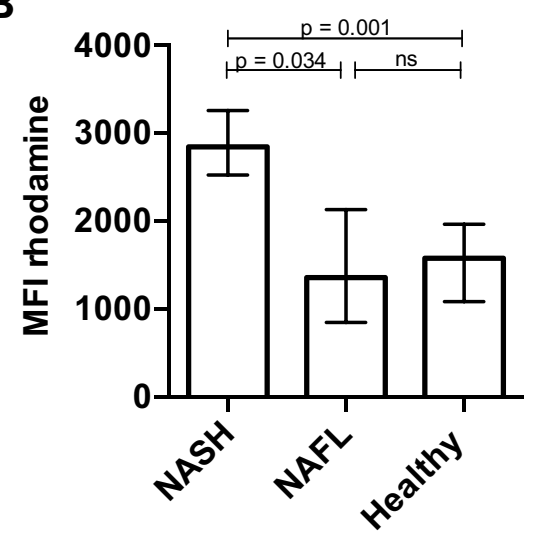

C

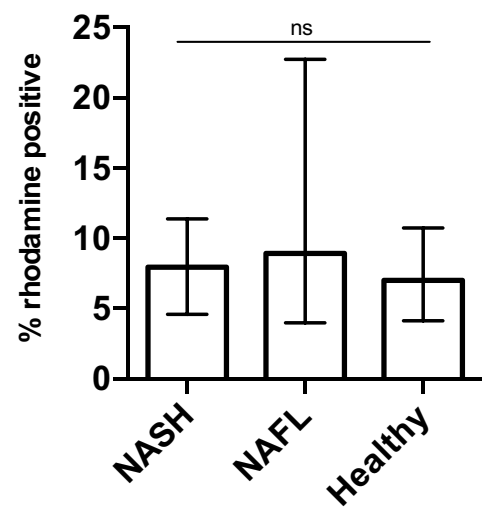

\section{Low oxidative burst}

D

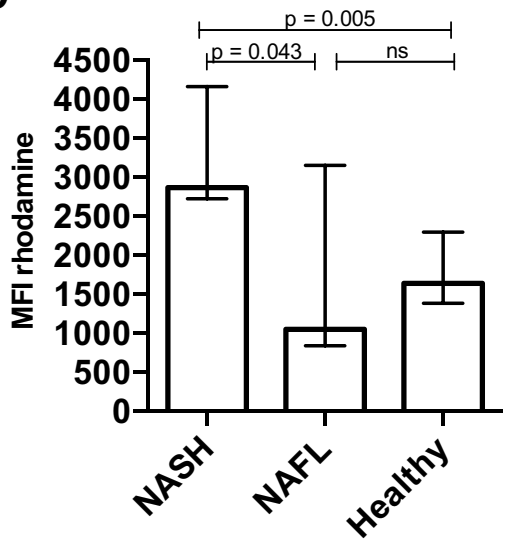

E

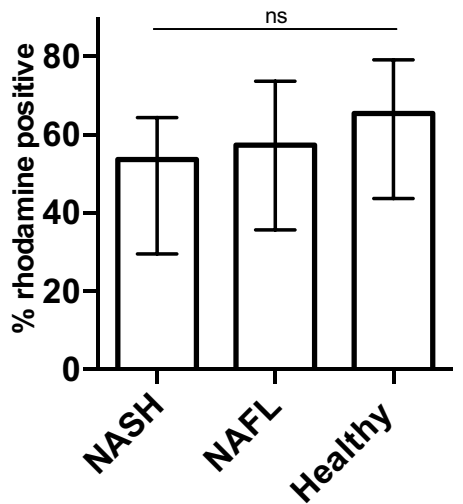

Figure 2 Functional priming of peripheral neutrophils in NASH patients. By flow cytometry, we measured (A) the frequency of IL-8-producing neutrophils in blood from patients with non-alcoholic steatohepatitis (NASH), non-alcoholic fatty liver (NAFL) and healthy subjects. Whole blood was incubated for 20 minutes at $37{ }^{\circ} \mathrm{C}$ and the conversion of dihydrorhodamine to rhodamine was measured without stimulation (B and $\mathbf{C}$ ) (spontaneous oxidative burst) and in the presence of formyl-methionyl-leucylphenylalanine (fMLP) (D and E) (low oxidative burst). The median fluorescent intensity (MFI) of rhodamine and the percentage of rhodamine-positive neutrophils are presented and compared among the groups (Kruskal-Wallis, rank sum test). Figures show median and interquartile range.

showed no sign of the NAFLD plasma increasing neutrophil activation or function. On the contrary, the NAFLD plasma incubated neutrophils displayed a slightly but systematically lower expression of CD62L compared with the neutrophils incubated with plasma from healthy persons, and the NAFL plasma in the same way decreased CD11b expression (Figure 3A and B). Functionally, the NASH patient plasma likewise reduced the frequency of IL-8-producing neutrophils and NAFL plasma also the frequencies of neutrophils producing an oxidative burst compared with plasma from healthy persons (Figure $3 \mathrm{C}$ and $\mathrm{D}$ ).

\section{Discussion}

Our study of NAFLD patients demonstrates an activation and functional priming of blood neutrophils and most markedly so in those patients with NASH. Moreover, we show that declining liver function and lack of glycemic control are associated with the degree of these findings. Unexpectedly, we found that plasma from NAFLD patients seemed to exert some de-activating effect on healthy neutrophils (Figure 4).

We report increased expression of CD62L and CD11b on blood neutrophils in NASH and a modest increase in CD11b across all NAFLD patients. The expression of these receptors reflects priming of the neutrophils for migration to tissues. This is different from patients with cirrhosis, acute-on-chronic liver failure, and acute liver failure where CD11b expression is reported to be unchanged while another activation receptor, CD16, is down-regulated, which we did not observe in our 
A

CD62L

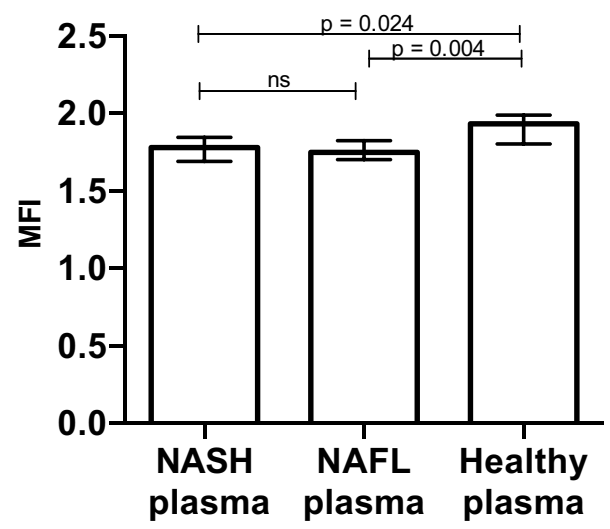

C Interleukin-8

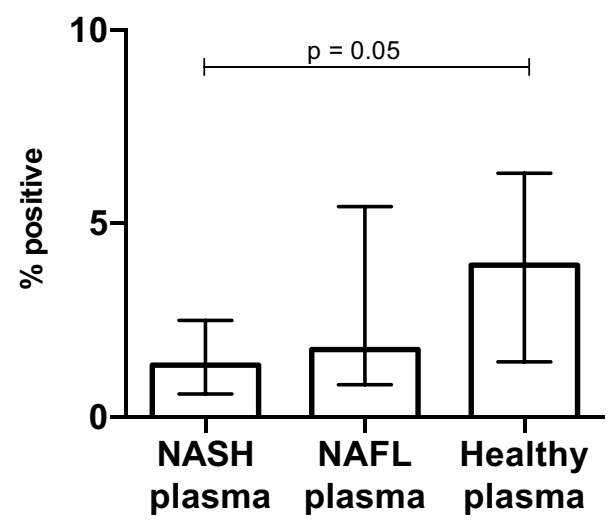

B

CD11b

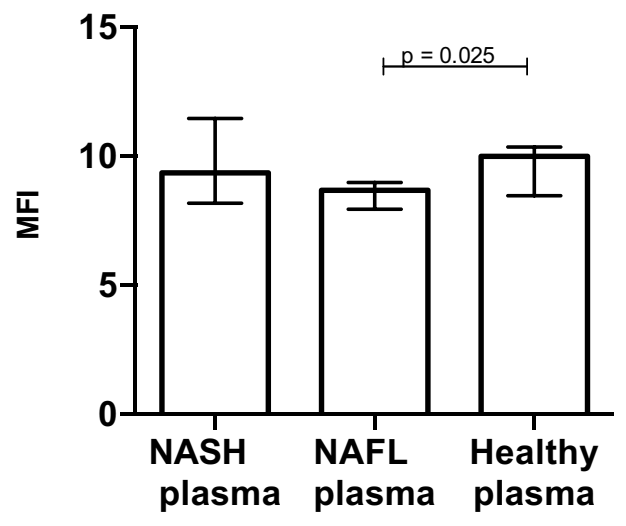

D Plasma-induced oxidative burst

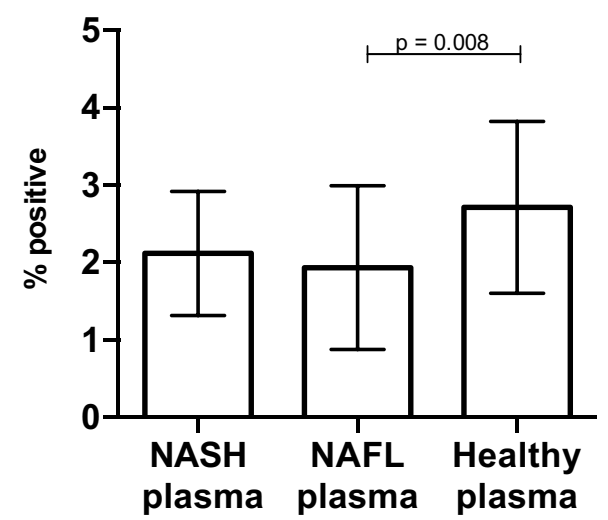

Figure 3 Plasma from NASH patients depress neutrophil activation and function. Neutrophils from a healthy volunteer were freshly isolated from peripheral blood and incubated with plasma from the study participants for 2 hours. The neutrophils were then harvested and the expression of the activation markers (A) CD62L and (B) CDIIb, (C) interleukin-8 and (D) dihydrorhodamine conversion to rhodamine, the oxidative burst, were measured by flow cytometry (Kruskal-Wallis, rank sum test). Figure displays median, interquartile range.

NAFLD patients. ${ }^{7,14}$ Thus, our finding may be specific to NAFLD, in line with experiments showing different migration receptors being involved in different types of liver injury. ${ }^{15,16}$ Our data may suggest that neutrophil migration, including into the liver, could be involved in the progression of NAFL to NASH. ${ }^{17}$ This is in accordance with other studies on liver biopsies showing histologically markedly more neutrophils and higher CD62L expression in NASH than in NAFL patients. ${ }^{6,18}$

The blood neutrophils from our NASH patients had an increased IL-8-production and a higher spontaneous- and low oxidative burst. The activated neutrophils described in $\mathrm{NASH}$ liver infiltrates are suggested to cause oxidative stress, liver injury, activation of macrophages and stellate cell collagen synthesis. ${ }^{6,19}$ Our study may add to the understanding of these mechanisms by indicating that NASH blood neutrophils, in addition to being prepared for migration, also are functionally primed. The neutrophil aggressiveness of NASH thus seems to be initiated already in the blood stream. The immediate explanation would be that factors present in the plasma of NASH patients are responsible, but as discussed below, this seems not to be the case, calling for alternative explanations.

When we exposed healthy blood neutrophils to plasma from NAFLD patients and healthy persons, we observed a modest but consistent downregulation by NAFLD 


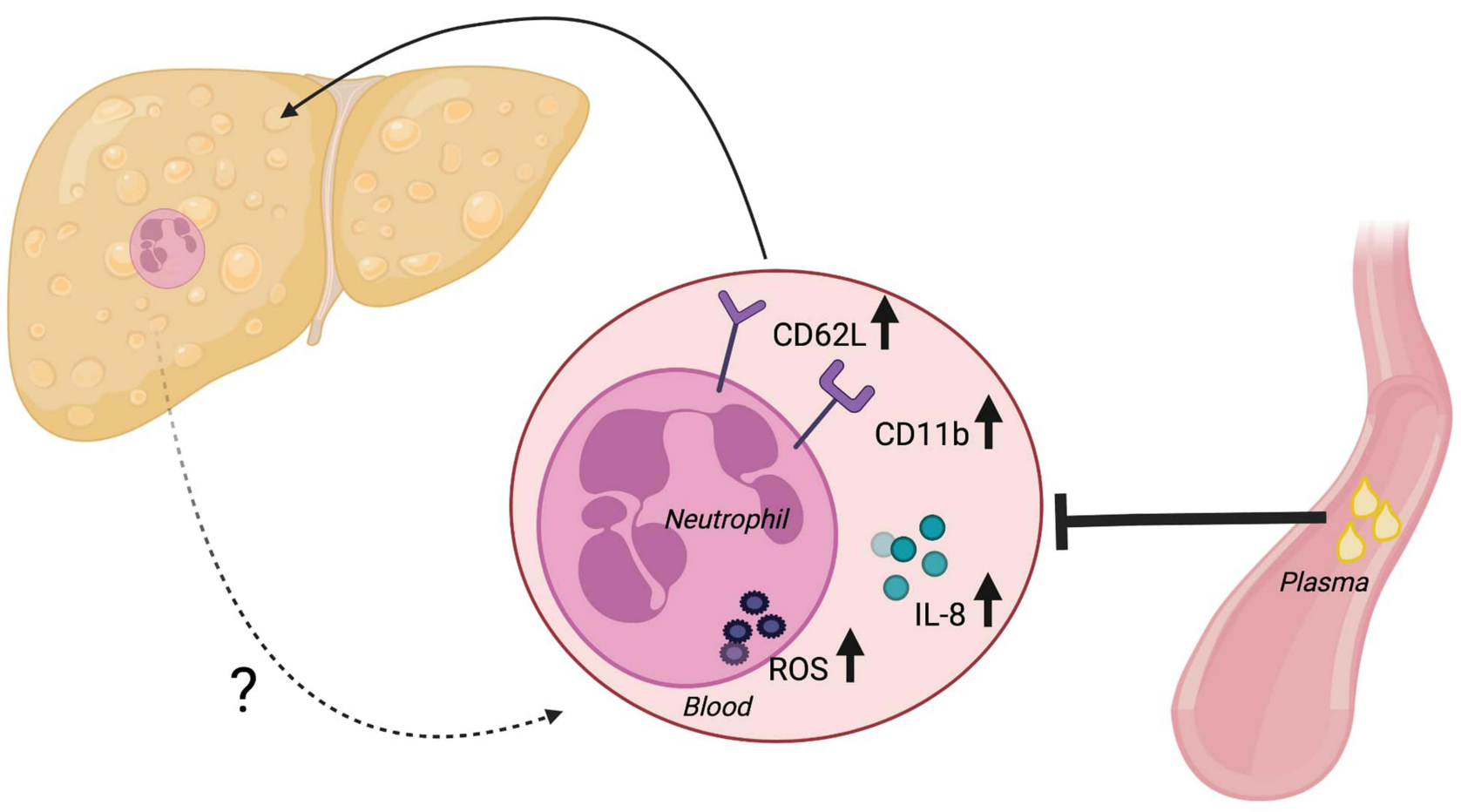

Figure 4 Summary of findings.

plasma of CD62L and CD11b and also of IL-8 and oxidative burst, which was the opposite to the phenotype of patient neutrophils. This finding was contrary to our a priori expectations based on findings in alcoholic hepatitis, where the blood neutrophil dysfunction was imitated in the healthy neutrophils incubated with patient plasma. ${ }^{9}$ This is also notable because we observed in the NAFLD patients an association between higher patient neutrophil CD11b expression and higher plasma triglycerides and glucose which might suggest that the plasma milieu could induce the activated neutrophil phenotype, in analogy with data linking glucose and fats to immune activation. $^{20-23}$ Although this might support that specific plasma factors could contribute to neutrophil priming, our data collectively suggest factors other than those present in plasma to be responsible for the aggressive blood neutrophil phenotype.

One possible alternative explanation would be that aggressive neutrophils to some extent exit the liver into the blood stream after becoming primed in the liver. Such "reverse transendothelial migration" as phenomenon actually occurs is currently debated. It breaks with the understanding that neutrophils undergo apoptosis after recruitment to sites of inflammation and thus move unidirectionally. ${ }^{24,25}$ Nonetheless, neutrophils described to have undergone reverse migration have a high oxidative burst, such as we describe in NASH blood neutrophils. ${ }^{26}$ In support of this possibility, the only identifiable factors distinguishing our NAFL from our NASH patients were indeed liver specific, ie, fibrosis and hepatocyte ballooning. This is also in line with the association we report between higher resting oxidative burst in patient neutrophils and worse metabolic liver function as measured by aminopyrine breath test.

Our study is limited by its small sample size and particularly the low number of NASH patients. Still, the unidirectional signals we obtained in our migration receptors and functional readouts and the consistent reversal of these findings by the plasma incubation, taken together corroborate our data.

Treatment options to halt the progression of NALFD are warranted, and biomarkers to predict progression are needed. As our data suggest blood neutrophils to be involved in disease progression, they may serve both as a marker of disease progression and be a treatment target. One of the currently available treatment options, which has shown some effect on NASH resolution is vitamin E. ${ }^{27}$ Vitamin $\mathrm{E}$ is an antioxidant and has in different experimental settings been demonstrated to lower neutrophil chemotaxis supporting the possibility of neutrophils as treatment targets. ${ }^{28-30}$ Future studies in this field may explore the hypothesis of neutrophil reverse migration from the liver to identify, which receptors may be 
involved. This could delineate possibilities to dampen the increased neutrophil activation and form the basis for targeting specific receptors as a therapeutic strategy.

In conclusion, blood neutrophils from NASH patients are primed to increased activation, function and migration. This does not happen in patients with only NAFL. The degree of this neutrophil priming is more marked with lower liver function and glycemic control. However, the aggressive neutrophil status was found not to depend on factors present in plasma from NASH patients, whereas such plasma to the contrary to some degree counteracted the NASH neutrophil changes we observe. This remains unexplained but if confirmed may suggest that some blood neutrophils in NASH patients might derive from the inflammatory intrahepatic environment and are exported to the blood. In this case, a vicious inter-organ circle seems to be established in which the liver selfperpetually maintains and aggravates the neutrophil aggressiveness that may be a mechanism for the fatty liver disease progression to fibrosis and ultimately cirrhosis.

\section{Abbreviations}

NAFLD, non-alcoholic fatty liver disease; NASH, nonalcoholic steatohepatitis; IL, interleukin; NAFL, nonalcoholic fatty liver; ROS, reactive oxygen species; PMA, phorbol 12-myristate 13-acetate; PDR, percentage dose recovery; fMLP, formyl-Met-Leu-Phe; MFI, mean fluorescence intensity.

\section{Data Sharing Statement}

The data supporting the results can be made available on request via e-mail to the corresponding author.

\section{Consent for Publication}

All authors have approved the final manuscript and authorship list and have consented to its publication.

\section{Funding}

This work was supported by Aase og Ejnar Danielsens Fond (grant no. 10-001533).

\section{Disclosure}

Professor Debbie Lindsay Shawcross reports grants from Norgine; personal fees from EnteroBiotix; received advisory board and lecture fees from Norgine; received lecture fees from Falk Pharma and Alfa Sigma; personal fees from Kaleido Biosciences and Shionogi, outside the submitted work. The authors have no other financial or non-financial conflicts of interest.

\section{References}

1. Adams LA, Lymp JF, St Sauver J, et al. The natural history of nonalcoholic fatty liver disease: a population-based cohort study. Gastroenterology. 2005;129(1):113-121. doi:10.1053/j. gastro.2005.04.014

2. Younossi ZM, Koenig AB, Abdelatif D, Fazel Y, Henry L, Wymer M. Global epidemiology of nonalcoholic fatty liver disease-metaanalytic assessment of prevalence, incidence, and outcomes. Hepatology. 2016;64(1):73-84. doi:10.1002/hep.28431

3. Vernon G, Baranova A, Younossi ZM. Systematic review: the epidemiology and natural history of non-alcoholic fatty liver disease and non-alcoholic steatohepatitis in adults. Aliment Pharmacol Ther. 2011;34(3):274-285. doi:10.1111/j.1365-2036.2011.04724.x

4. Gadd VL, Skoien R, Powell EE, et al. The portal inflammatory infiltrate and ductular reaction in human nonalcoholic fatty liver disease. Hepatology. 2014;59(4):1393-1405. doi:10.1002/hep.26937

5. Peng Y, Li Y, He Y, et al. The role of neutrophil to lymphocyte ratio for the assessment of liver fibrosis and cirrhosis: a systematic review. Expert Rev Gastroenterol Hepatol. 2018;12(5):503-513. doi:10.1080/ 17474124.2018.1463158

6. Rensen SS, Slaats Y, Nijhuis J, et al. Increased hepatic myeloperoxidase activity in obese subjects with nonalcoholic steatohepatitis. $\mathrm{Am}$ J Pathol. 2009;175(4):1473-1482. doi:10.2353/ajpath.2009.080999

7. Taylor NJ, Manakkat Vijay GK, Abeles RD, et al. The severity of circulating neutrophil dysfunction in patients with cirrhosis is associated with 90-day and 1-year mortality. Aliment Pharmacol Ther. 2014;40(6):705-715. doi:10.1111/apt.12886

8. Tritto G, Bechlis Z, Stadlbauer V, et al. Evidence of neutrophil functional defect despite inflammation in stable cirrhosis. J Hepatol. 2011;55(3):574-581. doi:10.1016/j.jhep.2010.11.034

9. Mookerjee RP, Stadlbauer V, Lidder S, et al. Neutrophil dysfunction in alcoholic hepatitis superimposed on cirrhosis is reversible and predicts the outcome. Hepatology. 2007;46(3):831-840. doi:10.1002/hep. 21737

10. Inzaugarat ME, Ferreyra Solari NE, Billordo LA, Abecasis R, Gadano AC, Chernavsky AC. Altered phenotype and functionality of circulating immune cells characterize adult patients with nonalcoholic steatohepatitis. J Clin Immunol. 2011;31(6):1120-1130. doi:10.1007/s10875-011-9571-1

11. Lykke Eriksen P, Sørensen M, Grønbæk H, Hamilton-Dutoit S, Vilstrup H, Thomsen KL. Non-alcoholic fatty liver disease causes dissociated changes in metabolic liver functions. Clin Res Hepatol Gastroenterol. 2019;43(5):551-560. doi:10.1016/j.clinre.2019.01.001

12. Støy S, Patel VC, Sturgeon JP, et al. Platelet-leucocyte aggregation is augmented in cirrhosis and further increased by platelet transfusion. Aliment Pharmacol Ther. 2018;47(10):1375-1386. doi:10.1111/apt.14600

13. Larsen FS, Schmidt LE, Bernsmeier C, et al. High-volume plasma exchange in patients with acute liver failure: an open randomised controlled trial. $J$ Hepatol. 2016;64(1):69-78. doi:10.1016/j. jhep.2015.08.018

14. Taylor NJ, Nishtala A, Manakkat Vijay GK, et al. Circulating neutrophil dysfunction in acute liver failure. Hepatology. 2013;57 (3):1142-1152. doi:10.1002/hep.26102

15. McDonald B, Jenne CN, Zhuo L, Kimata K, Kubes P. Kupffer cells and activation of endothelial TLR4 coordinate neutrophil adhesion within liver sinusoids during endotoxemia. Am J Physiol Gastrointest Liver Physiol. 2013;305(11):G797-806. doi:10.1152/ajpgi.00058.2013

16. Menezes GB, Lee WY, Zhou H, Waterhouse CC, Cara DC, Kubes P. Selective down-regulation of neutrophil Mac-1 in endotoxemic hepatic microcirculation via IL-10. J Immunol. 2009;183(11):7557-7568. doi:10.4049/jimmunol.0901786 
17. Diniz AB, Antunes MM, Lacerda VAS, et al. Imaging and immunometabolic phenotyping uncover changes in the hepatic immune response in the early phases of NAFLD. JHEP Rep. 2020;2 (4):100117. doi:10.1016/j.jhepr.2020.100117

18. Drescher HK, Schippers A, Rosenhain S, et al. L-selectin/CD62L is a key driver of non-alcoholic steatohepatitis in mice and men. Cells. 2020;9(5):1106. doi:10.3390/cells9051106

19. Ibusuki R, Uto H, Arima S, et al. Transgenic expression of human neutrophil peptide-1 enhances hepatic fibrosis in mice fed a choline-deficient, L-amino acid-defined diet. Liver Int. 2013;33 (10):1549-1556. doi:10.1111/liv.12203

20. van der Windt DJ, Sud V, Zhang H, et al. Neutrophil extracellular traps promote inflammation and development of hepatocellular carcinoma in nonalcoholic steatohepatitis. Hepatology. 2018;68 (4):1347-1360. doi:10.1002/hep.29914

21. Krenkel O, Hundertmark J, Abdallah AT, et al. Myeloid cells in liver and bone marrow acquire a functionally distinct inflammatory phenotype during obesity-related steatohepatitis. Gut. 2020;69 (3):551-563. doi:10.1136/gutjnl-2019-318382

22. Torres-Castro I, Arroyo-Camarena ÚD, Martínez-Reyes CP, et al. Human monocytes and macrophages undergo M1-type inflammatory polarization in response to high levels of glucose. Immunol Lett. 2016;176:81-89. doi:10.1016/j.imlet.2016.06.001

23. Xu X, Qi X, Shao Y, et al. High glucose induced-macrophage activation through TGF- $\beta$-activated kinase 1 signaling pathway. Inflamm Res. 2016;65(8):655-664. doi:10.1007/s00011-016-0948-8
24. Hamza B, Irimia D. Whole blood human neutrophil trafficking in a microfluidic model of infection and inflammation. Lab Chip. 2015;15(12):2625-2633. doi:10.1039/C5LC00245A

25. Hamza B, Wong E, Patel S, Cho H, Martel J, Irimia D. Retrotaxis of human neutrophils during mechanical confinement inside microfluidic channels. Integr Biol (Camb). 2014;6(2):175-183. doi:10.1039/ C3IB40175H

26. Buckley CD, Ross EA, McGettrick HM, et al. Identification of a phenotypically and functionally distinct population of long-lived neutrophils in a model of reverse endothelial migration. J Leukoc Biol. 2006;79(2):303-311. doi:10.1189/jlb.0905496

27. Sanyal AJ, Chalasani N, Kowdley KV, et al. Pioglitazone, vitamin E, or placebo for nonalcoholic steatohepatitis. $N$ Engl J Med. 2010;362 (18):1675-1685. doi:10.1056/NEJMoa0907929

28. Luostarinen R, Siegbahn A, Saldeen T. Effects of dietary supplementation with vitamin $\mathrm{E}$ on human neutrophil chemotaxis and generation of LTB4. Ups J Med Sci. 1991;96(2):103-111. doi:10.3109/ 03009739109179263

29. Rocksen D, Ekstrand-Hammarstrom B, Johansson L, Bucht A. Vitamin E reduces transendothelial migration of neutrophils and prevents lung injury in endotoxin-induced airway inflammation. $\mathrm{Am}$ J Respir Cell Mol Biol. 2003;28(2):199-207. doi:10.1165/rcmb.4899

30. Aoi W, Naito Y, Takanami Y, et al. Oxidative stress and delayed-onset muscle damage after exercise. Free Radic Biol Med. 2004;37(4):480-487. doi:10.1016/j.freeradbiomed.2004.05.008

\section{Publish your work in this journal}

Clinical and Experimental Gastroenterology is an international, peerreviewed, open access, online journal publishing original research, reports, editorials, reviews and commentaries on all aspects of gastroenterology in the clinic and laboratory. This journal is indexed on American Chemical Society's Chemical Abstracts Service (CAS)
The manuscript management system is completely online and includes a very quick and fair peer-review system, which is all easy to use. Visit http://www.dovepress.com/testimonials.php to read real quotes from published authors. 